\title{
Structural insights into DndE from Escherichia coli B7A involved in DNA phosphorothioation modification
}

Cell Research (2012) 22:1203-1206. doi:10.1038/cr.2012.66; published online 24 April 2012

\section{Dear Editor,}

DNA phosphorothioate (PT) modification, originally developed as an artificial tool to stabilize oligodeoxynucleotides against nuclease degradation [1], was recently found to be incorporated with sulfur into DNA backbone as a novel physiological variation by the five-gene $d n d$ cluster ( $d n d A-d n d E)$ products in a sequence- and stereospecific manner [2]. This PT modification causes the DNA degradation (Dnd) phenotype and is widespread and quantized in bacterial genomes, working as a part of a restriction modification system [3-5]. This modification can be specifically cleaved in vitro by type IV restriction endonuclease [6]. DndA works as a cysteine desulfurase and assembles DndC as a $4 \mathrm{Fe}-4 \mathrm{~S}$ cluster protein [7]. DndC possesses ATP pyrophosphatase activity $[8,9]$ and is predicted to have $3^{\prime}$-phosphoadenosine-5'phosphosulfate (PAPS) reductase activity, whereas DndB has homology to a group of transcriptional regulators $[10,11]$. DndD, known as SpfD in Pseudomonas fluorescens $\mathrm{Pf0}-1$, has ATPase activity possibly related to DNA structure alteration or nicking during PT incorporation $[5,12]$. Sequence identity $(46 \%)$ and similarity $(61 \%)$ to phosphoribosylaminoimidazole carboxylase (NCAIR synthetase) from Anabaena variabilis [11] suggest that DndE could be a NCAIR synthase analogue [13]. However, DndE may also act as a sulfotransferase due to a specific PAPS binding sequence AAVGK-TLLIHLHR contained in the C-terminus of DndE from Streptomyces lividans $\left(\mathrm{DndE}_{\text {strep }}\right)$ [10]. Therefore, the exact function of DndE remains unknown.

To address this, we over-expressed two DndE proteins from Escherichia Coli B7A (DndE $\mathrm{B}_{\mathrm{B} 7 \mathrm{~A}-\mathrm{full}}$ ) and Salmonella enterica $\left(\mathrm{DndE}_{\mathrm{Salm}-\mathrm{full}}\right)$, respectively (Supplementary information, Data S1), both having isoelectric point values close to 9.5. Sequence alignment indicates that they have high similarity except for several residues in their C-termini (Figure 1A). The sequence-selectivity of PT modification in both strains is a $d\left(G_{P S} A\right)$ or a $d\left(G_{P S} T\right)$ dinucleotide with an $R_{\mathrm{P}} \mathrm{PT}$ bond [5]. DndE $\mathrm{B}_{\mathrm{B} 7 \mathrm{~A} \text {-full }}$ is unstable, so we used its C-terminus-truncated form (aa 1-110,
$\left.\operatorname{DndE}_{\mathrm{B} 7 \mathrm{~A}-\mathrm{N} 110}\right)$ for crystallization. The aggregation states of DndE proteins were determined by dynamic light scattering (DLS) assay (Supplementary information, Data $\mathrm{S} 1$ ), indicating the formation of tetramers at the concentration of $200 \mu \mathrm{M}$. Two-dimensional NMR ${ }^{1} \mathrm{H}^{-15} \mathrm{~N}$ HSQC spectra on ${ }^{15} \mathrm{~N}$-labeled DndE $\mathrm{B}_{\mathrm{B} \text { A-N110 }}$ suggest that the Dn$\mathrm{dE}_{\mathrm{B} 7 \mathrm{~A}-\mathrm{N} 110}$ fragment in Tris buffer is more homogeneous (Supplementary information, Figure S1). The structure was determined using the single-wavelength anomalous dispersion technique, and refined to $2.5 \AA$ resolution with an $R_{\text {free }}$ factor of $24.2 \%$ and an $R$ factor of $20.1 \%$ (Supplementary information, Table S1).

The DndE $\mathrm{B}_{\mathrm{B} 7 \mathrm{~A}-\mathrm{N} 110}$ structure reveals a tetramer conformer (Figure 1B), consistent with the results from DLS assay. The tetramer adopts a quadrate fold, resembling a four-leaf clover of $62 \AA$ in both length and width and $32 \AA$ in height. It contains two kinds of dimers (A-E and A-M) with different stability resulted from different numbers of $\mathrm{H}$-bonds in the dimer interface (Figure $1 \mathrm{C}$ and 1D). Two clefts $\left(\sim 60^{\circ}\right.$ and $\left.\sim 90^{\circ}\right)$ are formed by the dimers (Figure 1E and 1F). Electrostatic surface analysis indicates that the cleft-containing side is more hydrophobic than its opposite side (Figure $1 \mathrm{G}$ and $1 \mathrm{H}$ ). The K20 side-chain in each monomer extends into the tetramer center, forming hydrogen bonds with G24 and/or G21 in the next monomer, producing a large positively charged hole (Figure 1B-1D and 1G). The four monomer structures are almost identical and can be superimposed with an root-mean-square deviation of $0.33 \AA$ over 59 backbone $\mathrm{C} \alpha$ atoms in the secondary structural region. Each monomer comprises five helices: $\mathrm{H} 1$ (aa 10-22), H2 (aa 27-39), H3 (aa 62-66), H4 (aa 70-77) and H5 (aa 86-102) (Figure 1A). Together with the $\mathrm{H} 2$ helix, a long flexible loop (aa 42-60) between the $\mathrm{H} 2$ and $\mathrm{H} 3$ helices encloses the H5 helix, where the residues R49, D50, S51 and K52 are not observable in some monomers.

Dali search indicates that $\operatorname{DndE}_{\mathrm{B} 7 \mathrm{~A}-\mathrm{N} 110}$ is a novel DNA-binding protein (Supplementary information, Figure S2). Thus, we performed DndE $\mathrm{B}_{\mathrm{B} 7 \mathrm{~A}-\mathrm{N} 110}$ binding studies using different DNA substrates in vitro, including dsDNA with or without a specific sequence ( $5^{\prime}$-GAAC-3') for 
A

$\alpha 1$

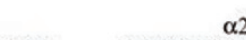

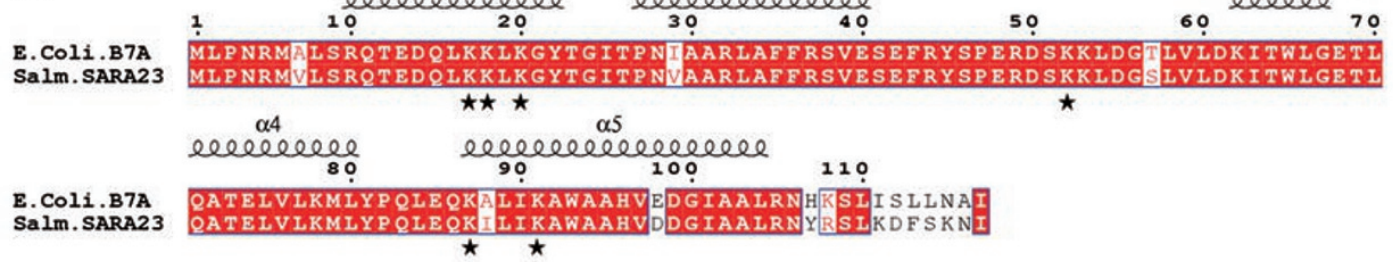

B

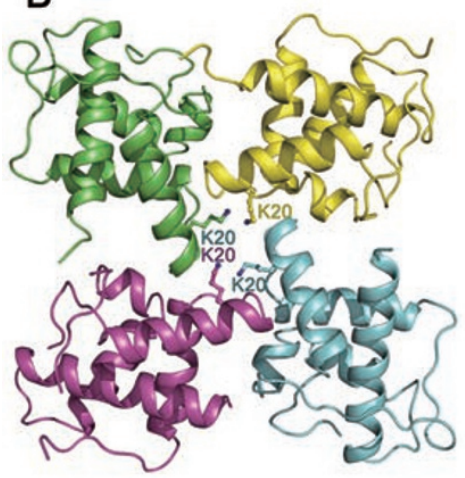

C

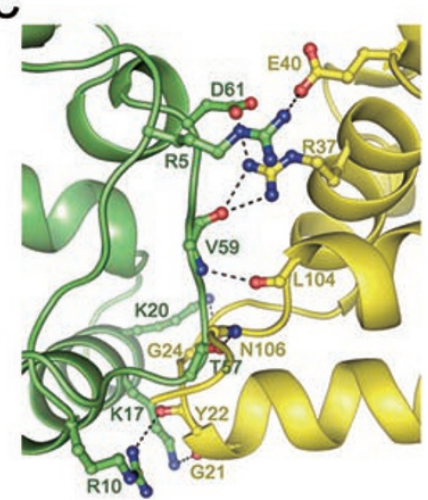

G

E

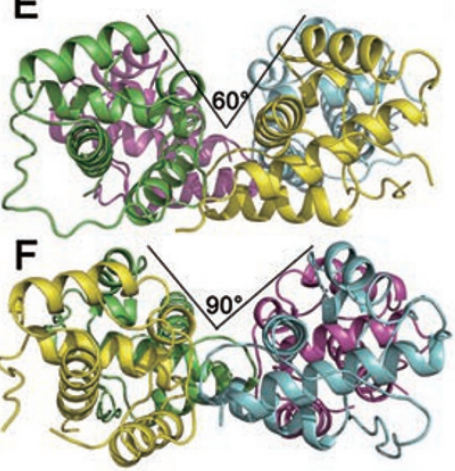

I

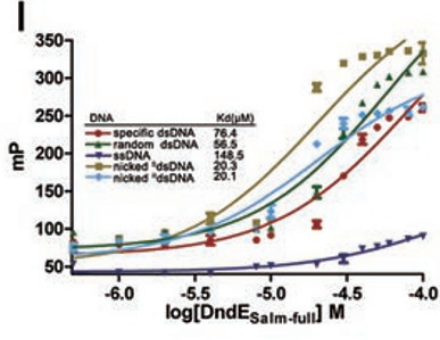

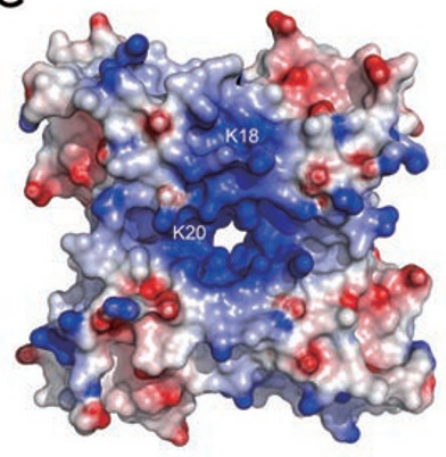

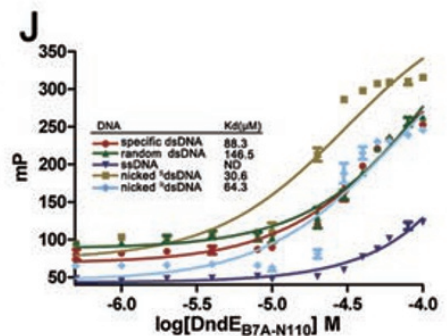

D

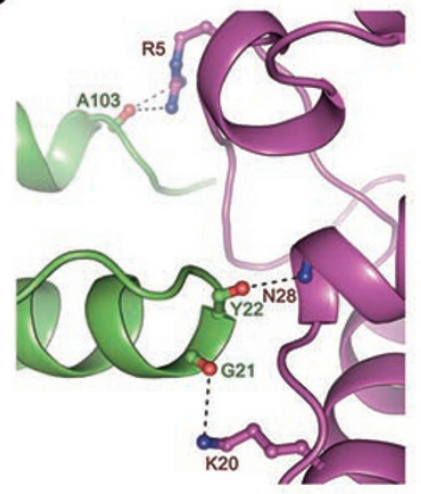

H
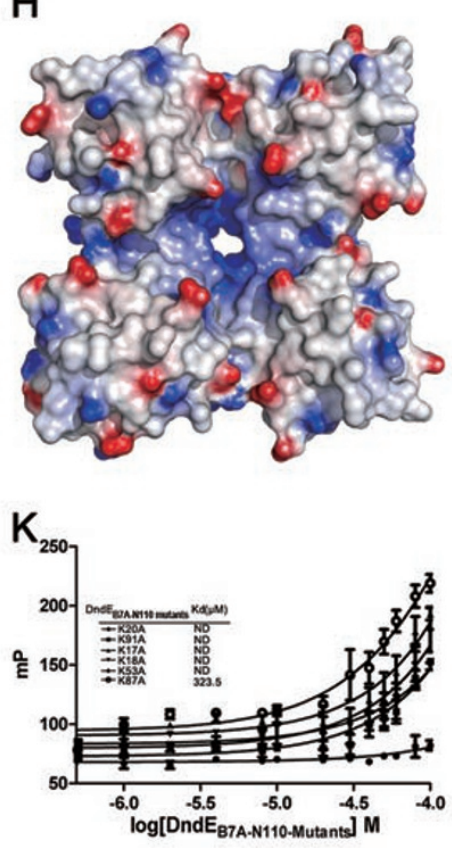

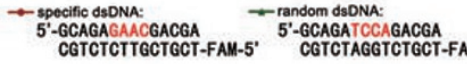

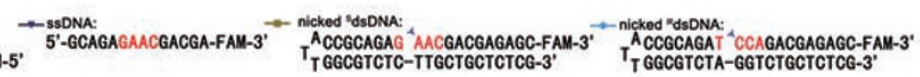

Figure 1 DndE structure analysis and fluorescence polarization assay for DNA-binding measurement. (A) Sequence alignment of DndE $E_{B 7 A-f u l l}$ and DndE $E_{\text {Salm-full }}$ with structural elements labeled on the top. (B) Ribbon diagram of DndE ${ }_{B 7 A-N 110}$ tetramer, monomer $\mathrm{A}$ is shown in green, $\mathrm{E}$ in purple, $\mathrm{C}$ in royal blue and $\mathrm{M}$ in yellow. (C, D) Interfaces between $\mathrm{A}-\mathrm{M}$ monomers and between A-E monomers. (E) Cleft formed in DndE $\mathrm{B}_{\mathrm{BA} \text {-N } 110}$ tetramer. (F) The view rotated by $90^{\circ}$ relative to the $\mathrm{x}$ axis from $\mathbf{E}$. (G) Electrostatic surface representation of $\mathrm{DndE}_{\mathrm{B} 7 \mathrm{~A}-\mathrm{N} 110}$ tetramer at the same orientation with $\mathbf{B}$. $(\mathbf{H})$ The view rotated by $90^{\circ}$ rela-

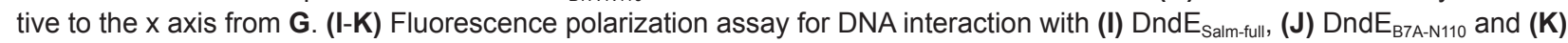
DndE $_{\mathrm{B} 7 \mathrm{~A}-\mathrm{N} 110}$ mutants, respectively. In the bottom, the sequences of DNA substrates were indicated, where the blur star represents phosphate. 
PT modification as found in E. coli B7A and Salmonella (specific dsDNA and random dsDNA in Figure 1). Since DndD is an ATPase with putative DNA-nicking actitvity $[5,12]$, we assumed that, before PT modification, DndD might hydrolyze the P-O bond between the G-A bases in PT modification sequence. Thus, two nicked dsDNA species were also tested (in one sequence, nicking is located between G-A bases in a specific sequence for PT modification, named as nicked ${ }^{\mathrm{S}} \mathrm{dsDNA}$; in the other sequence, nicking is designed between $\mathrm{T}-\mathrm{C}$ bases in a random sequence, denoted as nicked ${ }^{\mathrm{R}} \mathrm{dsDNA}$, as shown in Figure $1)$, as well as a sequence-specific single-stranded DNA (ssDNA in Figure 1). Among different DNA substrates, DndE $_{\text {Salm-full }}$ has the strongest binding affinity to nicked dsDNA (for both nicked ${ }^{\mathrm{S}}$ dsDNA and nicked ${ }^{\mathrm{R}}$ dsDNA, $\left.K_{\mathrm{D}} \sim 20 \mu \mathrm{M}\right)$, indicating that DndE might be a nicked dsDNA binding protein (Figure 1I), and that the specific -GAAC-sequence might not be relevant for nicked DNA binding. DndE $\mathrm{B}_{\mathrm{B} 7 \mathrm{~A}-\mathrm{N} 110}$ has a higher binding affinity to nicked ${ }^{\mathrm{S}} \mathrm{dsDNA}\left(K_{\mathrm{D}} \sim 30 \mu \mathrm{M}\right)$ than that to nicked ${ }^{\mathrm{R}}$ dsDNA $\left(K_{\mathrm{D}} \sim 60 \mu \mathrm{M}\right)$, specific dsDNA $\left(K_{\mathrm{D}} \sim 90 \mu \mathrm{M}\right)$, random dsDNA $\left(K_{\mathrm{D}} \sim 146.5 \mu \mathrm{M}\right)$, and specific ssDNA (binding is non-detectable) (Figure 1J, Supplementary information, Table S2), suggesting that DndE $\mathrm{B}_{\mathrm{B} 7 \mathrm{~A}-\mathrm{N} 110}$ also slightly prefers to bind with nicked dsDNA. Among the mutants where positively charged residues in the $\operatorname{DndE}_{\mathrm{B} 7 \mathrm{~A}-\mathrm{N} 110}$ surface including K17, K18, K20, K53, K87 and K91 are replaced by alanine, the K20A and K18A mutants showed significant decreases in binding affinities to specific dsDNA (K20A, $K_{\mathrm{D}} \sim 690 \mu \mathrm{M}$; K18A, non-detectable). All these variants showed much weaker $\left(\mathrm{K} 87 \mathrm{~A}, K_{\mathrm{D}}\right.$ $323 \mu \mathrm{M}$, decreased by 10 -fold) or non-detectable binding to nicked ${ }^{\mathrm{S}} \mathrm{dsDNA}$ (Figure 1K), further supporting that all these positively charged residues might be important for interaction with nicked dsDNA.

In addition, we measured the interaction of $\mathrm{DndE}_{\mathrm{B} 7 \mathrm{~A} \text { - }}$ N110 with PAPS, ATP or ADP using the isothermal titration calorimetry assay (Supplementary information, Figure S3). In contrast with the early prediction [10-11, 13], DndE $_{\text {B7A-N110 }}$ showed no binding affinities to any of them (Supplementary information, Figure S3), consistent with the fact that the DndE $\mathrm{B}_{\mathrm{B} 7 \mathrm{~A}-\mathrm{N} 110}$ structure includes neither a 5'-PSB motif nor a $3^{\prime}$-PB motif for PAPS and PAP binding [14], nor a glycine-rich P loop for ATP or ADP phosphate binding as seen in the NCAIR synthetase $[15,16]$.

Taken together, the crystal structure of DndE $\mathrm{B}_{\mathrm{B} 7 \mathrm{~A}-\mathrm{N} 110}$ reveals that DndE is neither a sulfotransferase nor a NCAIR synthase analogue, but a possible nicked dsDNA binding protein with a previously unrecognized fold. Thus, DNA nicking (probably by DndD [5, 12]) and nicked DNA binding by DndE might be essential for DNA PT modification.

\section{Acknowledgments}

This work was supported by National Basic Research Program of China (2009CB918600 and 2011CB966300), National Natural Science Foundation of China (20872169, 20905074 and 20921091), National New Drug Design Program from Ministry of Health of China (2011ZX09506), and Pujiang Talents Awards from Science and Technology Commission of Shanghai Municipality (08PJ14117). We thank Prof Jianhua He at the beamline BL17U at SSRF (China) for assistance in data collection.

Wei Hu ${ }^{1, *}$, Chengkun Wang ${ }^{1, *}$, Jingdan Liang ${ }^{2}$, Tianlong Zhang ${ }^{3}$, Zhongpei Hu ${ }^{1,4}$, Zhijun Wang ${ }^{2}$, Wenxian Lan ${ }^{1}$, Fang $\mathrm{Li}^{4}$, Houming $\mathrm{Wu}^{1}$, Jianping Ding ${ }^{3}$, Geng $\mathrm{Wu}^{2}$, Zixin Deng ${ }^{2}$, Chunyang $\mathrm{Cao}^{1}$

${ }^{1}$ State Key Laboratory of Bio-organic and Natural Product Chemistry, Shanghai Institute of Organic Chemistry, Chinese Academy of Sciences, 345 Lingling Road, Shanghai 200032, China; ${ }^{2}$ Laboratory of Microbial Metabolism, and School of Life Sciences and Biotechnology, Shanghai Jiao Tong University, Shanghai 200030, China; ${ }^{3}$ Institute of Biochemistry and Cell Biology, Shanghai Institutes for Biological Sciences, Shanghai 200031, China; ${ }^{4}$ Central China Normal University, Wuhan, Hubei 430079, China

*These two authors contributed equally to this work.

Correspondence: Chunyang Cao

Tel: 86-21-54925491; Fax: 86-21-64166128

E-mail: ccao@mail.sioc.ac.cn

\section{References}

1 Eckstein F, Gish G. Phosphorothioates in molecular biology. Trends Biochem Sci 1989; 14:97-100.

2 Wang L, Chen S, Xu T, et al. Phosphorothioation of DNA in bacteria by dnd genes. Nat Chem Biol 2007; 3:709-710.

3 Zhou X, Deng Z, Firmin JL, Hopwood DA, Kieser T. Site-specific degradation of Streptomyces lividans DNA during electrophoresis in buffers contaminated with ferrous iron. Nucleic Acids Res 1988; 16:4341-4352.

4 Dyson P, Evans M. Novel post-replicative DNA modification in Streptomyces: analysis of the preferred modification site of plasmid pIJ101. Nucleic Acids Res 1998; 26:1248-1253.

5 Wang L, Chen S, Vergin KL, et al. DNA phosphorothioation is widespread and quantized in bacterial genomes. Proc Natl Acad Sci USA 2011; 108:2963-2968.

6 Liu G, Ou H-Y, Wang T, et al. Cleavage of phosphorothioated DNA and methylated DNA by the type IV restriction endonucleases ScoMcrA. PloS Genetics 2010; 6:e1001253.

7 You D, Wang L, Yao F, Zhou X, Deng Z. A novel DNA modification by sulfur: DndA is a NifS-like cysteine desulfurase capable of assembling DndC as an iron-sulfur cluster protein in Streptomyces lividans. Biochemistry 2007; 46:6126-6133.

8 Lauhon CT, Kambampati R. The iscS gene in Escherichia coli is required for the biosynthesis of 4-Thiouridine, Thiamin, and NAD. J Biol Chem 2000; 275:20096-20103.

9 Mueller EG, Buck CJ, Palenchar PM, et al. Identification of 
a gene involved in the generation of 4-thiouridine in tRNA. Nucleic Acids Res 1998; 26:2606-2610.

$10 \mathrm{Ou}$ HY, He X, Shao Y, et al. dndDB: A database focused on phosphorothioation of the DNA backbone. PLoS One 2009; 4:e5132.

11 Zhou X, He X, Liang J, et al. A novel DNA modification by sulphur. Mol Microbiol 2005; 57:1428-1438.

12 Yao F, Xu T, Zhou X, Deng Z, You D. Functional analysis of $s p f D$ gene involved in DNA phosphorothioation in Pseudomonas fluorescens Pf0-1. FEBS Lett 2009; 583:729-733.

13 Nakamura Y, Kaneko T, Sato S, et al. Complete genome struc- ture of the thermophilic cyanobacterium Thermosynechococcus elongatus BP-1. DNA Res 2002; 9:123-130.

14 Kakuta Y, Pedersen LG, Perdersen LC, Negishi M. Conserved structural motifs in the sulfotransferase family. Trends Biochem Sci 1998; 23:129-130.

15 Thoden JB, Kappock TJ, Stubbe J, Holden HM. Three-dimensional structure of N5-carboxyaminoimidazole ribonucleotide synthetase: a member of the ATP grasp protein superfamily. Biochemistry 1999; 38:15480-15492.

16 Zhang Y, Morar M, Ealick SE. Structural biology of purine biosynthetic pathway. Cell Mol Life Sci 2008, 65:3699-3724.

(Supplementary information is linked to the online version of the paper on the Cell Research website.) 